\title{
BMJ Open Effect of an increased dosage of statins on spinal degenerative joint disease: a retrospective cohort study
}

\author{
Yuan-Yang Cheng, ${ }^{1,2,3}$ Chung-Lan Kao, ${ }^{2,4}$ Shih-Yi Lin,, ${ }^{3,5}$ Shin-Tsu Chang, ${ }^{1,6}$ \\ Tz-Shiang Wei, ${ }^{1}$ Shih-Ni Chang, ${ }^{7}$ Ching-Heng Lin $^{7,8,9}$
}

To cite: Cheng Y-Y, Kao C-L, Lin S-Y, et al. Effect of an increased dosage of statins on spinal degenerative joint disease: a retrospective cohort study. BMJ Open 2018;8:e017442. doi:10.1136/ bmjopen-2017-017442

- Prepublication history and additional material for this paper are available online. To view these files, please visit the journal online (http://dx.doi. org/10.1136/bmjopen-2017017442).

Received 24 April 2017

Revised 12 December 2017

Accepted 16 January 2018
Check for updates

For numbered affiliations see end of article.

Correspondence to Dr Ching-Heng Lin; epid@vghtc.gov.tw

\section{ABSTRACT}

Objectives It has been proven that statin can protect synovial joints from developing osteoarthritis through its anti-inflammatory effects. However, studies on the effect of statins on spinal degenerative joint diseases are few and limited to in vitro studies. Therefore, we investigated the relationship between the statin dosage and the development of spinal degenerative joint diseases.

Design A retrospective cohort study.

Setting Patients registered in Taiwan National Health Insurance Research Database.

Participants Patients aged 40-65 years old from 2001 to 2010 were included. Those who received statin treatment before 2001, were diagnosed with spinal degenerative joint diseases or received any spinal surgery before 2004 or had any spinal trauma before 2011 were excluded. A total of 7238 statin users and 164454 non-users were identified and followed up for the next 7 years to trace the development of spinal degenerative joint disease.

Outcome measures The incident rate of spinal degenerative joint diseases and HRs among the groups treated with different statin dosages.

Results A higher dosage of statins was associated with a significantly lower risk of developing spinal degenerative joint disease in patients with hypercholesterolaemia. Compared with the group receiving less than $5400 \mathrm{mg}$ of a statin, the HR of the 11900-28 000 mg group was 0.83 ( $95 \% \mathrm{Cl} 0.70$ to 0.99$)$, and that of the group receiving more than $28000 \mathrm{mg}$ was 0.81 (95\% $\mathrm{Cl} 0.68$ to 0.97 ). Results of subgroup analysis showed a significantly lower risk in men, those aged 50-59 years and those with a monthly income less than US $\$ 600$.

Conclusions Our study's findings clearly indicated that a higher dosage of statins can reduce the incidence of spinal degenerative joint disease in patients with hypercholesterolaemia, and it can be beneficial for people with a higher risk of spine degeneration.

\section{INTRODUCTION}

Low back pain is a common symptom that occurs in up to $80 \%$ of people during their lifetime, ${ }^{1}$ and it may significantly impact one's quality of life and work efficiency. Although the exact pathological source for low back pain cannot be definitely diagnosed in most patients, spinal degenerative joint

\section{Strengths and limitations of this study}

The Taiwan National Health Insurance Research Database is the largest and most representative medical database in Taiwan, which enabled us to follow the development of spinal degenerative joint diseases in all statin users without any loss of follow-up.

- The statin prescription record in our database may not be equal to the dosage patients actually took at home.

- The diagnosis of spinal degenerative joint diseases may be different among doctors in our database.

disease (DJD) is traditionally believed to be an important source of low back pain. ${ }^{2}$ Spinal DJD is the degenerative change in the threejoint complex of the spine (ie, one intervertebral disc and two posterior facet joints), which can be observed as decreased joint space and spur formation on plain radiographs. ${ }^{3}$ It is primarily caused by ageing-related disc change, repetitive microtrauma and axial loading stress. In addition, increased oxidative stress and inflammatory responses also play a significant role in the senescence mechanism of intervertebral disc degeneration. ${ }^{4}$

The 3-hydroxy-3-methylglutaryl coenzyme A reductase inhibitors, that is, statins, can inhibit the production of cholesterol by exerting its action on the rate-limiting step in cholesterol synthesis. ${ }^{5}$ Although they were initially developed to treat hyperlipidaemia, they are now widely used to treat patients with cerebrovascular and cardiovascular diseases because of their endothelial protective functions. ${ }^{6}$ Furthermore, it has been proven that statins mitigate inflammatory responses and oxidative stress by inhibiting a variety of proinflammatory cytokines. ${ }^{7}$ As previously mentioned, oxidative stress and inflammatory responses may contribute significantly to the pathogenesis of spinal DJD; therefore, it aroused our curiosity if statins could also be 
protective against the development of spinal DJD. Past studies have established the protective effect of statins on osteoarthritis of synovial joints, both in vitro ${ }^{89}$ and in vivo. ${ }^{10-12}$ In human epidemiological studies, statins have also been related to a decreased incidence of osteoarthritis. ${ }^{1314}$ It is worth noting that statin use was associated with more than a $50 \%$ reduction in the progression of knee osteoarthritis, but this effect could not be observed in hips in the Rotterdam study. ${ }^{14}$ In another human epidemiological study, statins were even related to an increased risk of developing hip osteoarthritis. ${ }^{15}$ Despite extensive research on the association between statin use and osteoarthritic synovial joints, studies on the association between statin use and spinal disc degeneration are few and limited to several in vivo studies. ${ }^{16-19}$ Therefore, we designed a retrospective cohort study to elucidate the effect of different dosages of statins on the epidemiological development of spinal DJD.

\section{MATERIALS AND METHODS The database}

This study analysed data from the Taiwan National Health Insurance Research Database (NHIRD), which was derived from the National Health Insurance programme in Taiwan since 1 March 1995. Nearly all citizens were included in this programme since birth, and data such as subjects' date of birth, sex, insured amount relating to their monthly income, living area and medical information, including the diagnoses of diseases by the International Classification of Diseases, ninth revision, clinical modification (ICD-9-CM) codes, medication dosages and durations prescribed and procedures received, were all recorded anonymously. The database we analysed was composed of systematically sampled one million people from all the beneficiaries in the year 2010 in the insurance programme, and there were no statistically significant differences in age, gender or healthcare costs between the sample group in the database and all beneficiaries under the programme. It is one of the largest medical databases in the world, and it has been used to analyse the epidemiology in Taiwan extensively. ${ }^{20}$ Therefore, we recruited our subjects from this database to obtain the most representative data in Taiwan to analyse the relationship between the dosage of the statin and incidence of spinal DJD.

\section{Case selection and study design}

Informed consent of our study subjects could not be obtained because all data were recorded anonymously in the NHIRD. We intentionally selected subjects aged between 40 and 65 to include the onset of spinal DJD, because the prevalence of radiographic change due to spinal DJD was higher in people aged more than 65 years in our database. Those who received any statin treatment before 1 January 2001, who had been diagnosed as having spinal DJD (ICD-9 codes: 721.0-721.4) before 1 January 2004, who received any surgical intervention of the spine
(ICD-9 codes: 03.01-03.99, 80.50-80.59 and 81.00-81.08) before 1 January 2004 and those who had been diagnosed as having spinal trauma (ICD-9 codes: 721.7, 805.0-805.9, 806.0-806.9, 839.00-839.59, 847.0-847.9, 952.0-952.9 and 953.0-953.9) at any time during the study period were excluded. For the remaining subjects, those who started to receive any type of statin from 1 January 2001 to 31 December 2003 were identified, and the summed dosage of all types of statins prescribed in the first 3 years was transformed into the equivalent simvastatin dose. ${ }^{21}$ The statin users were then divided into four groups according to the prescribed dosage of statins while maintaining a similar number of people per group. The dosages of statins used to divide the four groups were $5400 \mathrm{mg}, 11900 \mathrm{mg}$ and $28000 \mathrm{mg}$, which were designated as Q1, Q2 and Q3, respectively. Therefore, the four statin users groups were defined as statin prescribed $<5400 \mathrm{mg}$, $5400-11,900 \mathrm{mg}, 11900-28,000 \mathrm{mg}$ and $>28000 \mathrm{mg}$. On the other hand, those who did not receive any statin therapy from 1 January 2001 to 31 December 2003 were also included since 1 January 2001. The development of spinal DJD was followed over the next 7 years since 3 years after starting statin therapy in the statin users, and the same outcome was followed in the statin non-users since 1 January 2004. Because the cumulative dosage of statins were counted in the first 3 years since first prescription and the outcome was followed in the next 7 years only, there should be no collinearity between time and cumulative dosage of statins in our study, as shown in the online supplementary figure. To ensure that the diagnosis of spinal DJD was accurate, only those who had at least three times of coding 721.0-721.4 as the main outpatient diagnosis by different doctors after radiological examination were counted as having spinal DJD, and the first coding time was counted as the onset of spinal DJD. Other potential confounders, including age, sex the Charlson Comorbidity Index, monthly income and living area, were all recorded, and they were considered in further statistical adjustment during calculation of the HR.

\section{Statistical analysis}

We used Statistical Package for Social Sciences software package for Windows, V.17.0 (SPSS) for data analysis. Kruskal-Wallis test, $\chi^{2}$ tests and one-way analysis of variance (ANOVA) were used to assess statistical differences in age, sex, the Charlson Comorbidity Index, monthly income and living area among the statin users groups and the non-users. Kaplan-Meier survival analysis was then performed to generate the cumulative incidence of spinal DJD for the four groups of statin users and non-users. Cox proportional hazard regression analysis was performed to calculate the HR of the development of spinal DJD with reference to the group with a statin prescription less than $5400 \mathrm{mg}$ after adjusting for age, sex, the Charlson Comorbidity Index, monthly income and living area. Furthermore, stratified analysis of different age, sex and monthly income groups was performed to examine the HR of developing spinal DJD in patients 


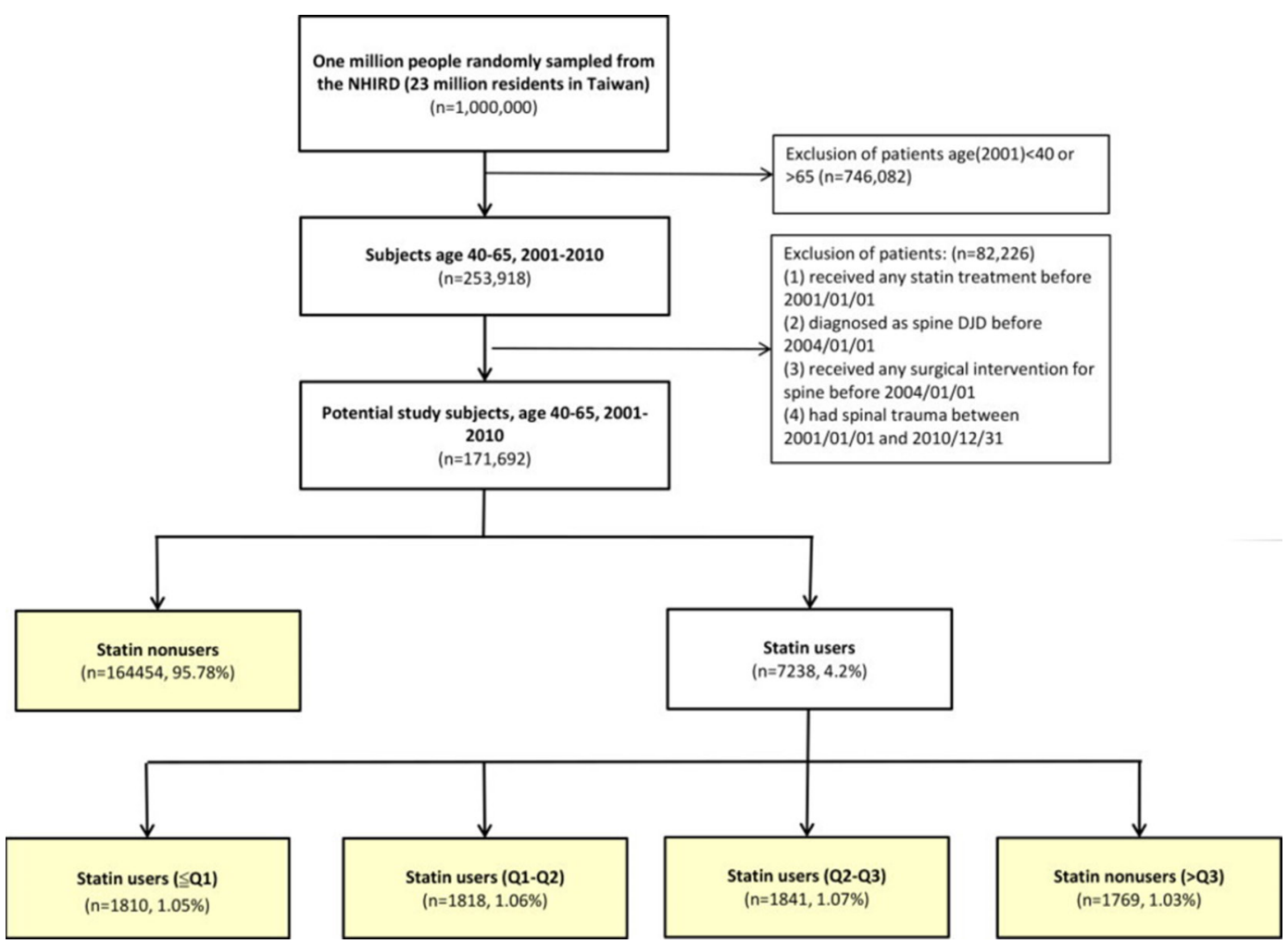

Figure 1 Study design. The flow chart illustrates how the statin users were selected and assigned to groups according to the different dosages of statins used in our study. Q1: Statin dosage of 5400 mg, Q2: statin dosage of 11900 mg, Q3: statin dosage of $28000 \mathrm{mg}$. DJD, degenerative joint disease; NHIRD, National Health Insurance Research Database.

with a cumulative dosage of statins more than Q1 with reference to less than $Q 1$. A $P$ value $<0.05$ was considered statistically significant in our study.

\section{RESULTS}

Overall, 7238 statin users and 164454 non-users were identified in our database, and the statin users were divided into four groups according to the dosage of statins in the first 3 years: group 1, <5400 mg; group 2, 5400-11 900 mg; group 3, 11900-28 000 mg; and group 4, >28000 mg. There were 1810 subjects in group 1, 1818 in group 2, 1841 in group 3 and 1769 in group 4. A flow chart of our subject selection and group assignment is shown in figure 1. A comparison of age, sex, the Charlson Comorbidity Index, monthly income and living area among the four groups and statin non-users is shown in online supplementary table 1 . Nearly all characteristics were significantly different among the five groups in Kruskal-Wallis test, $\chi^{2}$ tests and one-way ANOVA.

During the next 7 years of the follow-up period, 14633 subjects developed spinal DJD. The incident rates of spinal DJD were $1.25,2.57,2.4,2.19$ and 2.15 per 100 person-years in groups non-user, 1, 2, 3 and 4, respectively. The comparison of cumulative incidence of spinal DJD between statin users and non-users according to Kaplan-Meier analysis is shown in figure 2A, and that of the four statin users groups is shown in figure $2 \mathrm{~B}$. To elucidate the HR among the four groups, further Cox proportional hazard regression analysis was performed with reference to group 1 . The HR of the development of spinal DJD in group 2 was $0.93(0.79-1.10)$ versus group 1 , which did not achieve statistical significance. However, statistically significant higher HRs were noted in group 3 and group 4, which were 0.83 (95\% CI 0.70 to 0.99 ) and 0.81 (95\% CI 0.68 to 0.97 ), respectively. The HR of the statin non-users was 0.66 (95\% CI 0.58 to 0.74$)$. Detailed data are shown in table 1 .

To clarify the effect of the statin dosage in different age, sex and monthly income subgroups, stratified analysis of the HR was performed. As shown in figure 3, taking more than $5400 \mathrm{mg}$ of a statin in the first 3 years was shown to have a lower risk of developing spinal DJD within the 7-year follow-up period (HR 0.84, CI 0.74 to $0.97, \mathrm{P}=0.014)$. The protective effect was observed in all subgroups despite differences in age, sex and monthly income, and statistical significances were achieved in those aged between 50 and 59 years, men and those with an income less than US\$600 per month.

\section{DISCUSSION}

Our study's findings clearly showed a protective effect against the development of spinal DJD with a higher dosage of statins compared with a lower dosage of statins in patients with hypercholesterolaemia. Statin dosages more than $5400 \mathrm{mg}$ in the first 3 years significantly reduced the incidence of spinal DJD in the next 7 years. 
(A)

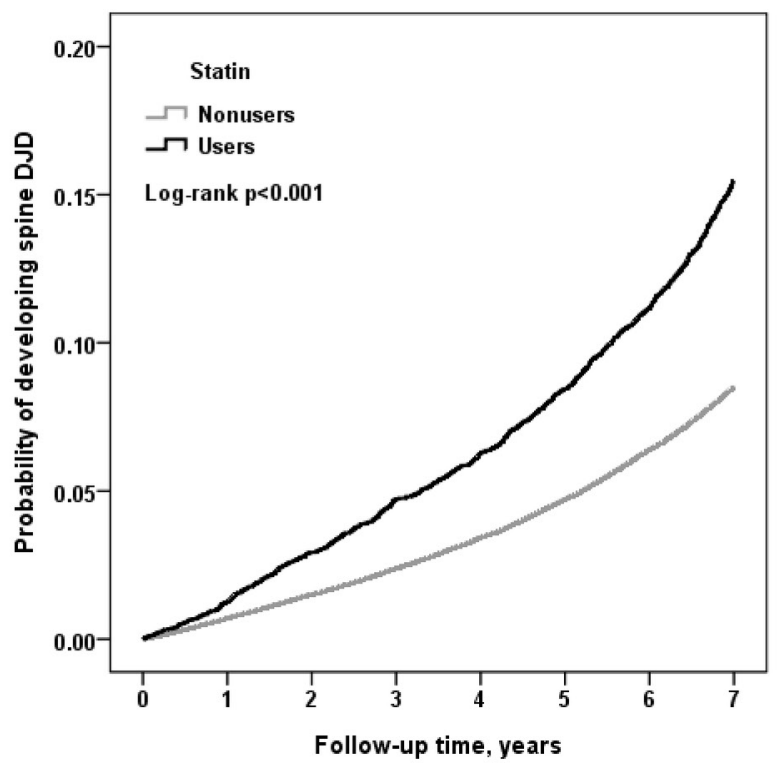

(B)

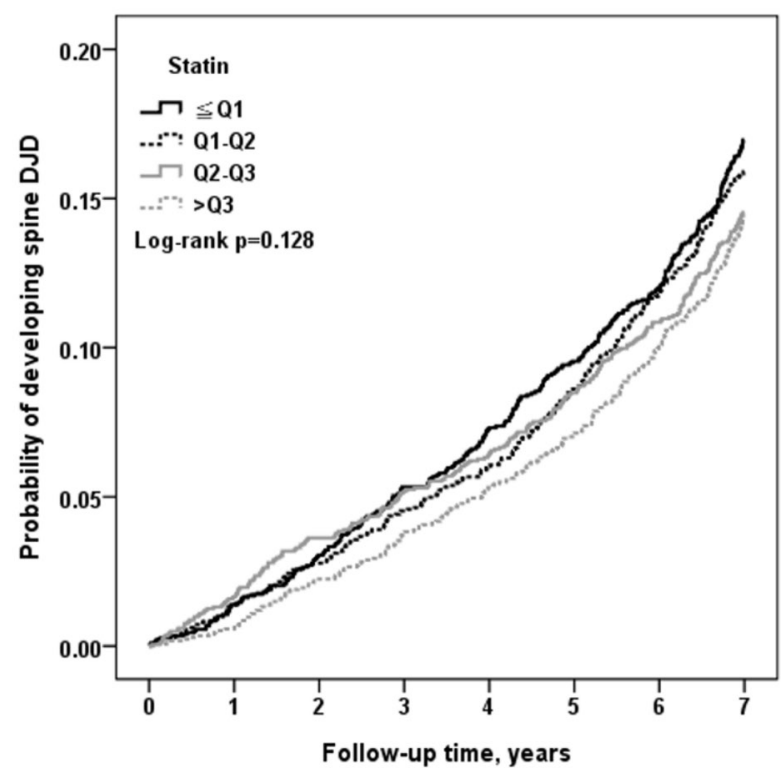

Figure 2 Cumulative incidence of spinal degenerative joint disease (DJD). (A) In each of the statin users and non-users, the cumulative incidence rates of spinal DJD are shown during the next 7 years of follow-up by the Kaplan-Meier method. (B) The cumulative incidence of spinal degenerative joints diseases are shown in each of the four statin users groups.

This is the first large-scale population-based cohort study on the relationship between the statin dosage and spinal DJD, and it can be corroborated by previous findings from in vivo studies.

$\mathrm{Hu}$ et $a l^{19}$ performed an interesting study on the effect of an intradiscal injection of lovastatin on iatrogenically induced spine disc degeneration in rats. They discovered that a higher concentration of lovastatin $(10 \mu \mathrm{M})$ produced more significant phenotypic disc repairs than lower concentrations $(0.1,1$ and $5 \mu \mathrm{M})$. In contrast, Than et $\mathrm{al}^{18}$ reported that degenerative discs injected with $5 \mathrm{mg}$ / $\mathrm{mL}$ of simvastatin in a hydrogel carrier demonstrated more improved radiographic and histological features compared with discs treated at higher doses (10 and $15 \mathrm{mg} / \mathrm{mL}$ ). Based on the aforementioned two studies, the exact dosage of a statin injected intradiscally that brought the most beneficial disc changes could not be determined since it was the lowest concentration in one study and the highest concentration in the other study. Nevertheless, they were still both impressive studies that demonstrated the dramatic effect of an intradiscal injection of a statin on disc degeneration. Statins are mostly taken up by the liver after oral administration and undergo first-pass metabolism, and their bioavailability may be greatly reduced in synovial joints and discs. Therefore, an important review article summarised that the protective effects on animal models of inflammatory and DJDs are less probative in clinical trials adopting oral administration of statins. ${ }^{22}$ Our study, however, still showed that the oral intake of a statin could also have a protective effect on the development of spinal DJD in patients with hypercholesterolaemia. Figure 2B clearly shows the dose-dependent response of a statin on the incidence of spinal DJD. Although the log-rank $\mathrm{P}$ value was 0.128 , which was not statistically significant, we still observed a significantly decreased HR in those taking 11900-28 000 mg (group 3 ) or more (group 4) compared with those taking less than $5400 \mathrm{mg}$ of a statin (group 1). As shown in figure 3, when we calculated the HR in those taking more than $5400 \mathrm{mg}$ of a statin (groups 2, 3 and 4 combined) versus group 1, it was still significantly decreased (HR 0.84, CI 0.74 to 0.97$)$. This result indicated that taking between $5400 \mathrm{mg}$ and $11900 \mathrm{mg}$ of a statin may be the cut-off point for the statin's beneficial effect on preventing the development of spinal DJD. However, the exact dosage should still be validated by further prospective, randomised, controlled studies in the future. Furthermore, alternative routes of administration of statin other than oral intake, such as intradiscal, intra-articular or transdermal, should be developed to increase the effectiveness of statin in joints diseases while preventing the adverse effects of high oral statin doses.

The complete mechanism underlying how statins can prevent discs from degeneration is still under investigation. Current evidence supports that statins can facilitate bone morphogenetic protein-2, aggrecan and type II collagen gene expression by inhibiting the production of mevalonate. ${ }^{16}$ As the nucleus pulposus of the disc is mainly composed of a mixture of aggrecan and type II collagen, ${ }^{23}$ statins could potentially reverse wear and tear due to ageing and repetitive microtrauma during everyday work. In addition to the anabolic effect of statins on the composition of the disc, statins could also prevent disc degeneration through its anti-inflammatory pathway. A past study showed that simvastatin can inhibit interleukin-6 and interleukin- 8 production in isolated chondrocytes and cartilage explants in a significant dose-dependent manner on stimulation by interleukin- $1 \beta$ and tumour 
Table 1 Comparison of the development of spinal DJD among patients taking different dosages of statins, age, gender, comorbidities and monthly income

\begin{tabular}{|c|c|c|c|c|c|}
\hline Statin dosage & No. of DJD events & Person-years & Incident rate† & Adjusted HR $(95 \% \mathrm{Cl})$ & P value \\
\hline Statin non-users & 13572 & 1084974 & 1.25 & 0.66 (0.58 to 0.74$)$ & $<0.001^{*}$ \\
\hline$\leq$ Q1‡ & 290 & 11289 & 2.57 & 1.00 & \\
\hline Q1-Q2‡ & 276 & 11518 & 2.40 & 0.93 (0.79 to 1.10$)$ & 0.382 \\
\hline Q2-Q3‡ & 255 & 11643 & 2.19 & 0.83 (0.70 to 0.99$)$ & $0.035^{*}$ \\
\hline >Q3‡ & 240 & 11166 & 2.15 & 0.81 (0.68 to 0.97$)$ & $0.019^{*}$ \\
\hline \multicolumn{6}{|l|}{ Age (years) } \\
\hline $40-49$ & 6374 & 666085 & 0.96 & 1.00 & \\
\hline $50-59$ & 5503 & 338763 & 1.62 & 1.59 (1.53 to 1.65$)$ & $<0.001^{*}$ \\
\hline $60-65$ & 2756 & 125741 & 2.19 & 1.99 (1.89 to 2.08 ) & $<0.001^{*}$ \\
\hline \multicolumn{6}{|l|}{ Gender } \\
\hline Female & 8670 & 548130 & 1.58 & 1.00 & \\
\hline Male & 5963 & 582460 & 1.02 & 0.66 (0.64 to 0.68$)$ & $<0.001^{*}$ \\
\hline \multicolumn{6}{|l|}{ Comorbidity } \\
\hline Diabetes mellitus & 1128 & 55434 & 2.03 & 0.79 (0.74 to 0.85$)$ & $<0.001^{*}$ \\
\hline Hyperlipidaemia & 1070 & 47835 & 2.24 & 1.14 (1.07 to 1.22$)$ & $<0.001^{*}$ \\
\hline $\begin{array}{l}\text { Cardiovascular } \\
\text { disease }\end{array}$ & 3632 & 165865 & 2.19 & 1.31 (1.26 to 1.37$)$ & $<0.001^{*}$ \\
\hline $\begin{array}{l}\text { Chronic kidney } \\
\text { disease }\end{array}$ & 74 & 3518 & 2.10 & 0.80 (0.63 to 1.01) & 0.061 \\
\hline \multicolumn{6}{|l|}{$\mathrm{CCl}$} \\
\hline 0 & 9103 & 861293 & 1.06 & 1.00 & \\
\hline 1 & 3303 & 178008 & 1.86 & 1.54 (1.47 to 1.60$)$ & $<0.001^{*}$ \\
\hline 2 & 1352 & 58422 & 2.31 & 1.83 (1.72 to 1.94$)$ & $<0.001^{*}$ \\
\hline$\geq 3$ & 875 & 32866 & 2.66 & 2.03 (1.88 to 2.20$)$ & $<0.001^{*}$ \\
\hline \multicolumn{6}{|l|}{$\begin{array}{l}\text { Monthly income } \\
\text { (US\$) }\end{array}$} \\
\hline$\leq 600$ & 7320 & 543565 & 1.35 & 1.00 & \\
\hline $600-1400$ & 2140 & 430205 & 0.50 & 0.99 (0.94 to 1.05$)$ & 0.707 \\
\hline$>1400$ & 3676 & 156820 & 2.34 & 0.89 (0.85 to 0.94$)$ & $<0.001^{*}$ \\
\hline
\end{tabular}

${ }^{*} \mathrm{P}<0.05$.

†Per 100 person-years.

$\mp Q 1$, statin dosage of $5400 \mathrm{mg}$; Q2, statin dosage of $11900 \mathrm{mg}$; Q3, statin dosage of $28000 \mathrm{mg}$.

$\mathrm{CCl}$, Charlson Comorbidity Index; DJD, degenerative joint disease.

necrosis factor- $\alpha .{ }^{9}$ Another study also showed that simvastatin can reduce the matrix metalloproteinase-3 level in interleukin-1ß-stimulated human chondrocyte culture. ${ }^{8}$ As an increased level of matrix metalloproteinases has been proven to be associated with disc degeneration, ${ }^{24} 25$ statins, which exert action on anti-inflammation and the reduction of matrix metalloproteinases, may also protect discs from degeneration.

Our study revealed that non-statin users had a lower incidence of spinal DJD. In Taiwan, the cost of statin prescription is reimbursed by National Health Insurance programme if a patient has hypercholesterolaemia. In the past literature, hypercholesterolaemia was evidenced to enhance the inflammatory status in human bodies, ${ }^{26}$ which is detrimental to chondrocytes in joints. ${ }^{27}$ Therefore, it could be expected that non-statin users, who had never been diagnosed as hypercholesterolaemia, had a lower incidence of spinal DJD in our study.

In the subgroup analysis, our study had an interesting finding that the protective effect of statins was significantly observed in men, those aged 50-59 years and those with a monthly income less than US\$600. In other subgroups, especially those who were women and aged $40-49$ years, a statin dosage more than $5400 \mathrm{mg}$ in the first 3 years had only a mildly decreased risk without statistical significance (HRs: women, 0.93; those aged 40-49 years, 0.95). A previous study showed that the protective effect of statins was not equal regarding sex, showing that statin therapy reduced the risk of coronary 
Variables

\begin{tabular}{l}
\hline Statin users \\
Age (years) \\
$40-49$ \\
$50-59$ \\
$60-65$ \\
Gender \\
Female \\
Male \\
Monthly income (USD) \\
$\leqq 600$ \\
$600-1,400$ \\
$>1,400$
\end{tabular}

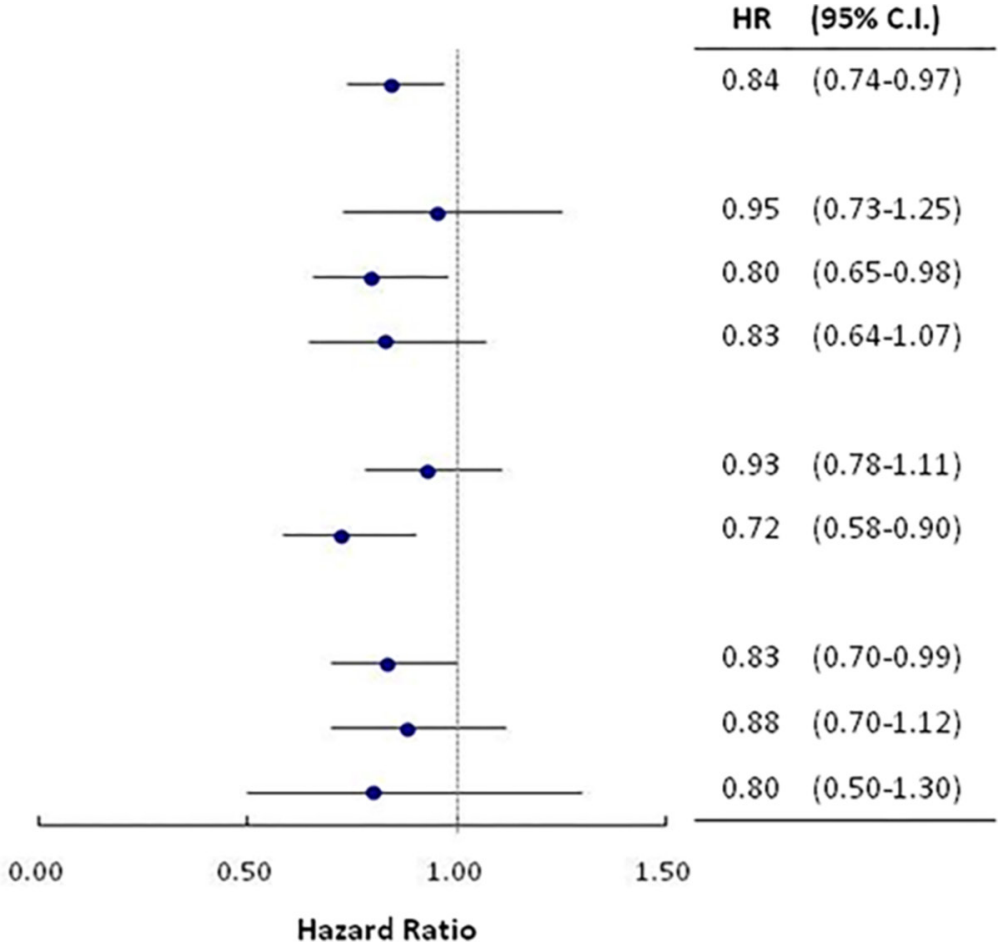

Figure 3 Results of the stratified subgroup analysis. The HR of the development of spinal degenerative joint disease in statin users with a dosage more than $5400 \mathrm{mg}$ was compared with those with a dosage less than $5400 \mathrm{mg}$ in different age, sex and monthly income subgroups. USD, US\$.

heart diseases in men without prior cardiovascular disease, but not in women. ${ }^{28}$ Coincidentally, our study also demonstrated a significant protective effect on the development of spinal DJD in men, but not in women. Past studies have also established strong evidence for the benefits of statins in the primary and secondary prevention of cardiovascular disease in those aged 40-75 years. ${ }^{29}$ For those less than 40 years old, statins are primarily reserved for treating severe hypercholesterolaemia only. ${ }^{30}$ Similarly, our study also showed only a borderline protective effect on the development of spinal DJD in the youngest group. Our study's findings further showed a significantly protective effect of statins in those with a monthly income less than US $\$ 600$, but the association between the beneficial effect of statins and one's socioeconomic status has never been explored before. Furthermore, the relationship between cardiovascular protection and preventative effects of statins on DJD deserves further investigation in the future.

Our study's findings clearly showed the protective effect of statins on the development of spinal DJD in patients with hypercholesterolaemia. One strength of our study is the population-based retrospective cohort design and use of the largest medical database in Taiwan, because it enabled us to follow the development of spinal DJD in all statin users without any loss of follow-up. Furthermore, the large sample size contributed to sufficient statistical power in elucidating the relationship between statin dosages and the incidence of spinal DJD. However, there are at least six limitations in our study. First, we could only record the prescription time and dosage of medications in our database; thus, we could not ensure whether the patients took these pills after they went home. Polypharmacy has been identified as a factor in poor medication adherence. ${ }^{31}$ We can assume that patients with a higher Charlson Comorbidity Index who took a higher dosage of a statin in our study had a lower medication compliance. Even so, they still had a lower incidence of spinal DJD, although the actual dosage of the statin they took may be less than that recorded. Another issue was that the statin dosage in our study was the summed dose in the first 3 years, and we could not identify whether the patients took all the drugs in the first few months or took them averagely during the 3 years, which may influence the homogeneity in each groups in our study. Second, the degree of degenerative changes shown on a plain radiograph was required to establish a diagnosis of spinal DJD, which may have been different among doctors. For example, a radiograph considered to show mild degenerative changes of the spine for one doctor may still be deemed as within normal range for another doctor. To prevent arbitrary diagnoses made by one doctor, a diagnosis of spinal DJD was counted only if it was listed as a main diagnosis three times at different outpatient visits in our study. Third, some patients may acquire spinal DJD without seeking medical help, such as those with lower socioeconomic status, older age or with other comorbidities. However, Taiwan is a small island with easily assessed medical services. Furthermore, the existence of National Health Insurance enables patients to get medical help with extremely low costs. Therefore, this 
limitation could be of less concern in our study using the NHIRD. We further statistically adjusted for age, sex, the Charlson Comorbidity Index, monthly income and living area during Cox regression analysis to reduce the impact of this limitation. Fourth, the time patients seek medical help may not represent the onset of spinal DJD. The study results would be strengthened if all patients were clinically assessed on the onset of spinal DJD. Fifth, the major risk factor of spinal DJD is considered to be axial loading during work and daily activities. However, the information of occupation and working properties could not be obtained in our database, and we could only take monthly income and living area into statistical adjustment instead. Finally, the subjects we analysed in our study were mainly Taiwanese; thus, the study results may not be extrapolated to other racial groups. However, our findings can still serve as a valuable reference for physicians of Asian countries.

\section{CONCLUSIONS}

A higher dosage of statins can reduce the incidence of spinal DJD in patients with hypercholesterolaemia according to the results of our study. Thus, physicians have another clinically significant reason, in addition to cardiovascular benefits, to recommend their patients to take statins at a higher dosage under the consideration of the possible side effects such as liver and muscle damage, elevated blood sugar, memory loss or confusions. Further prospective case-controlled studies should be done to validate our study results in the future.

\section{Author affiliations}

${ }^{1}$ Department of Physical Medicine and Rehabilitation, Taichung Veterans General Hospital, Taichung, Taiwan

${ }^{2}$ School of Medicine, National Yang-Ming University, Taipei, Taiwan

${ }^{3}$ Center for Geriatrics and Gerontology, Taichung Veterans General Hospital, Taichung, Taiwan

${ }^{4}$ Department of Physical Medicine and Rehabilitation, Taipei Veterans General Hospital, Taipei, Taiwan

${ }^{5}$ Division of Endocrinology and Metabolism, Department of Internal Medicine, Taichung Veterans General Hospital, Taichung, Taiwan

${ }^{6}$ Department of Physical Medicine and Rehabilitation, School of Medicine, National Defense Medical Center, Taipei, Taiwan

${ }^{7}$ Department of Medical Research, Taichung Veterans General Hospital, Taichung, Taiwan

${ }^{8}$ Department of Public Health, College of Medicine, Fu Jen Catholic University, New Taipei City, Taiwan

${ }^{9}$ Department of Health Care Management, National Taipei University of Nursing and Health Sciences, Taipei, Taiwan

Acknowledgements We would like to thank Editage (www.editage.com) for the English language editing. We would also like to thank the Healthcare Service Research Center (HSRC) of Taichung Veterans General Hospital for statistical support.

Contributors $\mathrm{Y}-\mathrm{YC}$ and $\mathrm{C}-\mathrm{HL}$ contributed in the study design, manuscript writing and final approval of the version to be published. C-LK and S-YL contributed in the data acquisition, analysis, critically revising and final approval of the version to be published. S-TC, S-NC and T-SW contributed in the data interpretation, critically revising and final approval of the version to be published. C-HL is the correspondent author and takes responsibility for all aspects of the reliability of the data presented and interpretation.
Funding This work was supported by Taichung Veterans General Hospital (Grant number TCVGH-NHRI10505).

Competing interests None declared.

Patient consent Detail has been removed from this casedescription/these case descriptions to ensure anonymity. The editors andreviewers have seen the detailed information available and are satisfied thatthe information backs up the case the authors are making.

Ethics approval The Institutional Review Committee of Taichung Veterans General Hospital in Taiwan (No. CE13152).

Provenance and peer review Not commissioned; externally peer reviewed. Data sharing statement No additional data are available.

Open Access This is an Open Access article distributed in accordance with the Creative Commons Attribution Non Commercial (CC BY-NC 4.0) license, which permits others to distribute, remix, adapt, build upon this work non-commercially, and license their derivative works on different terms, provided the original work is properly cited and the use is non-commercial. See: http://creativecommons.org/ licenses/by-nc/4.0/

(c) Article author(s) (or their employer(s) unless otherwise stated in the text of the article) 2018. All rights reserved. No commercial use is permitted unless otherwise expressly granted.

\section{REFERENCES}

1. Patrick N, Emanski E, Knaub MA. Acute and Chronic Low Back Pain. Med Clin North Am 2016;100:169-81.

2. Cheung KM, Karppinen J, Chan D, et al. Prevalence and pattern of lumbar magnetic resonance imaging changes in a population study of one thousand forty-three individuals. Spine 2009;34:934-40.

3. Yong-Hing K, Kirkaldy-Willis WH. The pathophysiology of degenerative disease of the lumbar spine. Orthop Clin North Am 1983;14:491-504.

4. Wang F, Cai F, Shi R, et al. Aging and age related stresses: a senescence mechanism of intervertebral disc degeneration. Osteoarthritis Cartilage 2016;24:398-408.

5. Gazzerro P, Proto MC, Gangemi G, et al. Pharmacological actions of statins: a critical appraisal in the management of cancer. Pharmacol Rev 2012;64:102-46.

6. Wolfrum S, Jensen KS, Liao JK. Endothelium-dependent effects of statins. Arterioscler Thromb Vasc Biol 2003;23:729-36.

7. Tousoulis D, Psarros C, Demosthenous M, et al. Innate and adaptive inflammation as a therapeutic target in vascular disease: the emerging role of statins. J Am Coll Cardiol 2014;63:2491-502.

8. Lazzerini PE, Capecchi PL, Nerucci F, et al. Simvastatin reduces MMP-3 level in interleukin 1 beta stimulated human chondrocyte culture. Ann Rheum Dis 2004:63:867-9.

9. Dombrecht EJ, Van Offel JF, Bridts $\mathrm{CH}$, et al. Influence of simvastatin on the production of pro-inflammatory cytokines and nitric oxide by activated human chondrocytes. Clin Exp Rheumatol 2007;25:534-9.

10. Akasaki $\mathrm{Y}$, Matsuda S, Nakayama K, et al. Mevastatin reduces cartilage degradation in rabbit experimental osteoarthritis through inhibition of synovial inflammation. Osteoarthritis Cartilage 2009;17:235-43.

11. Barsante MM, Roffê E, Yokoro CM, et al. Anti-inflammatory and analgesic effects of atorvastatin in a rat model of adjuvant-induced arthritis. Eur J Pharmacol 2005;516:282-9.

12. Bayyurt $\mathrm{S}$, Küçükalp $\mathrm{A}$, Bilgen $\mathrm{MS}$, et al. The chondroprotective effects of intraarticular application of statin in osteoarthritis: An experimental study. Indian J Orthop 2015;49:665-71.

13. Kadam UT, Blagojevic M, Belcher J. Statin use and clinical osteoarthritis in the general population: a longitudinal study. J Gen Intern Med 2013;28:943-9.

14. Clockaerts S, Van Osch GJ, Bastiaansen-Jenniskens YM, et al. Statin use is associated with reduced incidence and progression of knee osteoarthritis in the Rotterdam study. Ann Rheum Dis 2012;71:642-7.

15. Beattie MS, Lane NE, Hung YY, et al. Association of statin use and development and progression of hip osteoarthritis in elderly women. $J$ Rheumatol 2005;32:106-10.

16. Zhang H, Lin CY. Simvastatin stimulates chondrogenic phenotype of intervertebral disc cells partially through BMP-2 pathway. Spine 2008;33:E525-E531.

17. Zhang $\mathrm{H}$, Wang L, Park JB, et al. Intradiscal injection of simvastatin retards progression of intervertebral disc degeneration induced by stab injury. Arthritis Res Ther 2009;11:R172. 
18. Than KD, Rahman SU, Wang L, et al. Intradiscal injection of simvastatin results in radiologic, histologic, and genetic evidence of disc regeneration in a rat model of degenerative disc disease. Spine $J$ 2014:14:1017-28.

19. Hu MH, Yang KC, Chen YJ, et al. Lovastatin prevents discographyassociated degeneration and maintains the functional morphology of intervertebral discs. Spine J 2014;14:2459-66.

20. Hsing AW, loannidis JP. Nationwide population science: lessons from the Taiwan National Health Insurance Research Database. JAMA Intern Med 2015;175:1527-9.

21. Weng TC, Yang YH, Lin SJ, et al. A systematic review and metaanalysis on the therapeutic equivalence of statins. J Clin Pharm Ther 2010;35:139-51.

22. Lazzerini PE, Capecchi PL, Selvi E, et al. Statins and the joint: multiple targets for a global protection? Semin Arthritis Rheum 2011;40:430-46.

23. Mwale F, Roughley P, Antoniou J. Distinction between the extracellular matrix of the nucleus pulposus and hyaline cartilage: a requisite for tissue engineering of intervertebral disc. Eur Cell Mater 2004;8:58-64. discussion 63-4.

24. Weiler C, Nerlich AG, Zipperer J, et al. 2002 SSE Award Competition in Basic Science: expression of major matrix metalloproteinases is associated with intervertebral disc degradation and resorption. Eur Spine J 2002;11:308-20.

25. Shen B, Melrose J, Ghosh P, et al. Induction of matrix metalloproteinase-2 and -3 activity in ovine nucleus pulposus cells grown in three-dimensional agarose gel culture by interleukin1beta: a potential pathway of disc degeneration. Eur Spine $J$ 2003; $12: 66-75$

26. da Silva Pereira R, Tatsch E, Bochi GV, et al. Assessment of oxidative, inflammatory, and fibrinolytic biomarkers and DNA strand breakage in hypercholesterolemia. Inflammation 2013;36:869-77.

27. Simonaro CM, Haskins ME, Schuchman EH. Articular chondrocytes from animals with a dermatan sulfate storage disease undergo a high rate of apoptosis and release nitric oxide and inflammatory cytokines: a possible mechanism underlying degenerative joint disease in the mucopolysaccharidoses. Laboratory Investigation 2001;81:1319-28.

28. Petretta M, Costanzo P, Perrone-Filardi P, et al. Impact of gender in primary prevention of coronary heart disease with statin therapy: A meta-analysis. Int J Cardiol 2010;138:25-31.

29. Stone NJ, Robinson JG, Lichtenstein AH, et al. ACC/AHA guideline on the treatment of blood cholesterol to reduce atherosclerotic cardiovascular risk in adults: a report of the American College of Cardiology/American Heart Association Task Force on Practice Guidelines. J Am Coll Cardiol 2014;63:2889.

30. Stone NJ, Turin A, Spitz JA, et al. Statin therapy across the lifespan: evidence in major age groups. Expert Rev Cardiovasc Ther 2016;14:341-66.

31. Dury R. Medication non-compliance in older patients: a multifactorial problem. Br J Community Nurs 2013;18:383-5. 